\title{
Childhood Environments and Cytomegalovirus Serostatus and Reactivation in Adults
}

\author{
Denise Janicki-Deverts ${ }^{a}$, Sheldon Cohen ${ }^{a}$, William J. Doyle ${ }^{b}$, Anna L. Marsland ${ }^{\mathrm{c}}$, and Jos A. \\ Bosch $^{\mathrm{d}, \mathrm{e}, \mathrm{f}}$
}

aDepartment of Psychology, Carnegie Mellon University, Pittsburgh, PA 15213, USA bDepartment of Otolaryngology/ENT, Children's Hospital of Pittsburgh, Pittsburgh, PA 15224, USA 'Department of Psychology, University of Pittsburgh, Pittsburgh, PA 15260, USA dDepartment of Clinical Psychology, University of Amsterdam, Amsterdam, The Netherlands ${ }^{\text {Mannheim Institute }}$ of Public Health, Social and Preventive Medicine (MIPH), University of Heidelberg, Mannheim, Germany ${ }^{f}$ Department of Health and Human Performance, University of Houston, Houston, TX, USA

\section{Abstract}

Childhood adversity, defined in terms of material hardship or physical or emotional maltreatment has been associated with risk for infection with cytomegalovirus (CMV) among children and adolescents, and with CMV reactivation in children and adults. The present study examined whether different dimensions of childhood experience- those pertaining to socioeconomic status (SES), physical environment, or family relationships, relate differentially to CMV serostatus and reactivation during adulthood. Participants were 140 healthy adults, aged $18-55$ years (41\% female; $64 \%$ white). Childhood environments were assessed retrospectively and included family SES (parental housing tenure); childhood neighborhood environment (urban residence; physical conditions; safety; and social atmosphere); residential exposures (parental smoking and physical condition of home); and family relationships (parental divorce; warmth; harmony; dysfunction; parental bonding). Approximately 39\% $(n=53)$ of participants were CMV+. In individual analyses controlling for age, sex, race, body mass, current adult SES and smoking status, fewer years of parental home ownership, having a parent who smoked, and living in a poorly maintained or unsafe neighborhood each were associated with greater odds of infection with CMV. By comparison, in individual analyses limited to CMV+ participants, less family warmth, less harmony, greater dysfunction, and suboptimal parental bonding each were related to higher antibody levels, independent of the aforementioned covariates. Findings were not attributable to current adult perceptions of psychological stress or relative levels of emotional stability. These

(C) 2014 Elsevier Inc. All rights reserved.

Corresponding author: Denise Janicki-Deverts, Department of Psychology, Carnegie Mellon University, Pittsburgh, PA 15213, Telephone: 412-268-3295, djanicki@andrew.cmu.edu.

Publisher's Disclaimer: This is a PDF file of an unedited manuscript that has been accepted for publication. As a service to our customers we are providing this early version of the manuscript. The manuscript will undergo copyediting, typesetting, and review of the resulting proof before it is published in its final citable form. Please note that during the production process errors may be discovered which could affect the content, and all legal disclaimers that apply to the journal pertain. 
results suggest that different types of childhood adversity may be associated with differential effects on CMV infection and latency.

\section{Keywords}

childhood environments; cytomegalovirus; latent virus; psychological stress; reactivation

\section{Introduction}

Exposures to unfavorable physical and psychosocial environments during early life reliably have been associated with increased risk of morbidity and premature mortality during adulthood (Cohen, Janicki-Deverts, Chen, \& Matthews, 2010; Miller, Chen, \& Parker, 2011). One pathway through which childhood experiences have been hypothesized to influence adult health involves long-term dysregulation of the immune system. Several studies have shown childhood adversity, defined in terms of material hardship or physical or emotional maltreatment, to be associated with poor immune response in adults. Much of this work has examined circulating markers of inflammation as the outcome. Early socioeconomic disadvantage, for example, has been associated with relatively elevated concentrations of circulating pro-inflammatory cytokines (Carroll, Cohen, \& Marsland, 2011) and C-reactive protein (CRP; Phillips, Marsland, Flory, Muldoon, Cohen, \& Manuck, 2009; Pollitt, Kaufman, Rose, Diez-Roux, Zeng, \& Heiss, 2007) at midlife. Lower socioeconomic status (SES) during childhood and adolescence also has been associated with increased likelihood of developing acute upper respiratory illness-a process partly attributable to the activity of pro-inflammatory cytokines, following exposure to a virus that causes the common cold (Cohen, Doyle, Turner, Alper, \& Skoner, 2004; Cohen et al., 2013). Similarly, accumulated exposure to parental abuse and/or neglect during early life, has been related to elevated levels of interleukin [IL]-6 among both healthy adults (Danese, Parlante, Caspi, Taylor, \& Poulton, 2007) and women with breast cancer (Janusek, Tell, Albuquerque, \& Mathews, 2013). Early adversity has also been found to interact with current adult life stress, amplifying the detrimental effects of current stress on inflammation (Kiecolt-Glaser, Gouin, Weng, Malarkey, Beversdorf, \& Glaser, 2011) and local response to a basal cell carcinoma tumor (Fagundes, et al., 2012).

Another aspect of adult immunity that appears to be influenced by unfavorable exposures during early life involves the processes implicated in establishing resistance to reactivation of latent viruses. Among young adult (mean age 29 years) participants in the National Longitudinal Study on Adolescent Health (Add Health) who had detectable antibody to Epstein Bar Virus (EBV), lower parental SES assessed during adolescence was associated with higher blood antibody levels (Slopen, McLaughlin, Dunn, \& Koenen, 2013). However, a re-analysis of these same data using a different cut off of antibody levels for defining serostatus failed to find an association between adversity and antibody levels (Dowd, Palermo, Chyu, Adam, \& McDade, 2013). By comparison, in a sample of female breast cancer survivors who were seropositive for EBV and/or cytomegalovirus (CMV), higher scores on a retrospective summary measure of childhood adversity (including for example death of a parent, parental marital discord, lacking a close relationship with at least one 
adult) were associated with elevated levels of antibody against both viruses (Fagundes, Glaser, Malarkey, \& Kiecolt-Glaser, 2013). Finally, among adolescents who were seropositive for herpes simplex virus type 1 (HSV-1), institutional rearing and physical abuse during childhood each were associated with elevated HSV-1 antibody levels relative to demographically similar controls (Shirtcliff, Coe, \& Pollak, 2009).

Although provocative, these studies have limitations. The two analyses of the Add Health data produced inconsistent results, and the association between childhood adversity and antibody levels in the Fagundes et al. (2013) sample was based on a female clinical population. The Shirtcliff et al. (2009) sample, too, was drawn from two special populations (children adopted from Eastern European and Chinese orphanages; adolescents with a history of physical abuse). Moreover, although the adolescent study examined whether the association of early adversity with antibody levels could be explained by higher rates of infection among the maltreated (groups did not differ in infection rates), neither of the two adult studies examined whether the same factors associated with elevated antibody levels also predict seropositivity.

In the present study, we use data from a sample of healthy adult male and female volunteers to elaborate upon the existing research on childhood adversity and latent virus infection and reactivation. Specifically, we focus on identifying which dimensions of childhood experience-those pertaining to socioeconomic, physical, or familial environments, are most crucial for individuals' initial risk for viral infection and which are associated with longterm effects on immunologic resistance to subsequent reactivation. We also examine whether any found associations are independent of the effects of adult SES. We examine CMV serostatus and antibody levels as our model of latent virus infection and reactivation. CMV is prevalent throughout the United States, with infection rates ranging from about $35 \%$ among young children to $90 \%$ among older adults (Staras, Dollard, Radford, Flanders, Pass, \& Cannon, 2006). Until recently CMV infection and non-clinical reactivation were thought to be without symptoms among healthy adults, and associated with detrimental effects only in vulnerable populations. However, this view has changed, with CMV infection increasingly being considered a public health concern. Among adults, CMV seropositivity has been associated with increased mortality risk both in community samples with broad age ranges (Simanek, Dowd, Pawelec, Melzer, A., \& Aiello, 2011; Gkrania-Klotsas, Langenberg, Sharp, Luben, Khaw, \& Wareham, 2013) and in healthy older adults (Savva, et al., 2013). Moreover, prenatal infection with CMV has been associated with increased risk of infant mortality and long-term disability (Ross, Dollard, Victor, Sumartojo, \& Cannon, 2006). Elevated CMV antibody levels, as well, have been linked to increased mortality in older adults, and, in all age groups, to immune dysregulation characteristic of accelerated aging of the immune system (immunosenescence) including impaired vaccination responses (McElhaney et al., 2012; Turner et al., 2013), increased inflammation (Freeman, 2009; Qiu, Straat, Rahbar, Wan, Soderberg-Naucler, \& Haeggstrom, 2008), impaired ability to proliferate to mitogens (Chidrawar, et al., 2009), reduced telomere length (van de Berg et al., 2010), and reduced telomerase activity (Dowd et al., 2013). Thus, gaining a better understanding into early life factors affecting risk for infection and reactivation of this virus may have important implications for public health and may contribute to understanding of the role of early life determinants of healthy aging (Nikolich-Zugich, 2008). 


\section{Materials and Methods}

\subsection{Participants}

Participants were drawn from a sample of 212 healthy volunteers aged 18-55 years from the greater Pittsburgh, PA area who were recruited by newspaper and posted advertisement to participate in a study of psychological and behavioral influences on resistance to the common cold. Each was paid $\$ 1,000$ for their participation in the parent study. The 140 men and women included in the present analyses comprised all participants with sufficient stored blood available for assessment of CMV antibodies. The study was conducted between 2007 and 2011 and was approved by the Institutional Review Boards of both Carnegie Mellon University and the University of Pittsburgh and all participants provided signed informed consent.

\subsection{Procedures}

Volunteers presenting for possible enrollment in the parent study underwent medical screenings and were excluded if they had been either treated in the past year or hospitalized in the past 5 years for psychiatric illness; had a history of major nasal or otologic surgery, respiratory disorders, or cardiovascular disease; had abnormal urinalysis, complete blood count, or blood enzymes; were currently pregnant or lactating; tested seropositive for human immunodeficiency virus; or were regularly taking medication other than birth control. The parent study was designed to examine social, psychological, behavioral, and biological factors that influence participants' susceptibility to developing upper respiratory illness following experimental exposure to a common cold virus (rhinovirus [RV] 39; Cohen et al., 2013). Thus, specific serum neutralizing antibody against RV39 was assessed at screening, and volunteers also were excluded if their RV39 antibody levels were $>4 \mathrm{IU}$, which is considered protective against this virus.

All data included in this article were collected during the baseline period (before viralchallenge) of the parent study. Sera for CMV antibody assessments were obtained by standard venipuncture into serum separator tubes (see Cohen et al., 2013). Samples were spun for 10 minutes at $3500 \mathrm{rpm}$. Serum was placed into labeled 2-ml microcentrifuge tubes, and then stored at $-70^{\circ} \mathrm{C}$ until shipped on dry ice to the University of Birmingham School of Sport and Exercise Sciences, Behavioural Immunology Laboratory (Birmingham, United Kingdom) for CMV antibody assay.

Self-reported adult demographic data and current perceived stress were collected by questionnaire at the time of screening for the parent study and the entry physical exam, respectively. Self-report questionnaires assessing childhood environments and adult personality were administered on the same day as the blood draw for CMV antibody assessment.

\subsection{Childhood environments}

2.3.1. Childhood neighborhood environment-The Places You've Lived Interview is comprised of 15 items asking respondents to describe the place(s) in which they lived during childhood and adolescence (Available at www.psy.cmu.edu:16080/ scohen/; Cohen, 
2010a). The 15 items are repeated (as a set) three times, with reference to ages 5, 10, and 15 years. The choice of items to include in the PLI was informed by research demonstrating detrimental effects of negative physical and psychological environmental exposures during childhood and adolescence on adult health outcomes (Cohen et al., 2010). The first item asks participants to provide the name of the town or city (and state) that they lived in at the reference age; and the second to identify whether that area was a city, suburb, small town, or rural community. The remaining items can be divided into 3 scales that describe the physical conditions, safety, and social aspects of the respondent's childhood neighborhood environment. The physical conditions scale was comprised of 6 items $(\mathrm{yes}=1, \mathrm{no}=0$ ) inquiring about the presence of traffic, noise, graffiti, litter, and poor street maintenance that were summed to create a total score. The safety scale was comprised of 3 items that asked participants to rate the frequency with which their street was considered safe, they observed violent acts, and saw people using drugs or drinking alcohol. Response options ranged from $1=$ all the time to $4=$ never , and were reversed for the latter two items so that higher values would indicate less safe conditions. The safety scale score was computed by taking the mean of the 3 component items. The social environment scale was comprised of 4 items that asked about the friendliness of the neighborhood, the presence of responsible neighbors, the presence of friends, and how often participants (at the reference ages) were allowed to play outside. Response options were the same as for the items comprising the safety scale, and were scored so that higher values indicated less favorable social conditions. The social environment scale score was computed by taking the mean of the 4 component items. For the present analyses, summary scores were created for each dimension of neighborhood environment by aggregating individual scale scores across the three reference ages. Specifically, a total urban residence score was computed by summing the number of reports of having lived in a city (range, $0-3$ ); overall neighborhood physical condition (Cronbach's $a=0.76-0.82$ ), safety (Cronbach's $a=0.64-0.67$ ), and social environment (Cronbach's $a=$ $0.71-0.76)$ scores were derived by averaging across the three reference ages.

2.3.2. Family socioeconomic status (SES)—Childhood SES was estimated based on parental housing tenure when participants where children and adolescents. Specifically, for each year between ages 1 and 18 years, participants were asked whether their parent(s) owned the family home (measure available at www.psy.cmu.edu/ scohen; Cohen, 2010b). Responses were summed to create a continuous parental home ownership variable that ranged from 0 to 18. An advantage of using home ownership as a retrospective marker of childhood SES is that people are confidently able to recall whether their parents owned the family home from fairly early childhood (Cohen et al., 2004).

2.3.3. Residential exposures-Two potential sources of toxic exposures within the home were examined: parental smoking and the physical condition of the home itself. In reference to each of three ages during childhood $(5,10$, and 15 years-old), participants were asked to recall the frequency with which each of their parents smoked, and the condition of the house in which they lived (very well kept/good condition, moderately well kept, fair condition, or poor condition). From these data, two dichotomous variables were created indicating whether either of the participant's parents were smokers (i.e., participant endorsed any response option other than never for either parent at any of the three reference ages); 
and if they rated their childhood home as being in either fair or poor condition at any of the three reference ages.

\subsubsection{Family interpersonal environment}

2.3.4.1. Parental divorce: Participants were asked whether their parents ever separated or divorced and their age at the time the separation/divorce took place. A dichotomous variable was created indicating those participants who were 18 years of age or younger when their parents separated or divorced.

2.3.4.2. Emotional atmosphere: Using a 4-point frequency scale $(1=$ never to $4=$ all the time) participants reported how often their parents showed them affection, praised them, hugged them, showed interest in their school work, and how often their family laughed together (Cohen, 2010b). A family warmth index was created by taking the mean of participant responses to each of these 5 items. To accommodate random missing data, means were computed for all participants with data for at least 3 of the 5 items. Emotional atmosphere was assessed for ages 5, 10, and 15 years. An overall family warmth variable was computed by averaging the index scores across the three reference ages (Cronbach's a $=0.89-0.90)$.

2.3.4.3. Family harmony: General childhood family harmony, or the extent to which interpersonal relationships among family members were amicable and supportive was assessed using three 5-item subscales from the Family Environment Scale (FES; Moos \& Moos, 1994) that are thought to comprise the interpersonal relationships dimension of family environment: cohesion (family members helped and supported one another), expressiveness (family members often told each other about personal problems), and conflict (family fought a lot). Response options for all items were presented in a 5-point Likert format, ranging from 1 = strongly disagree to $5=$ strongly agree, and prefaced by a reference to "When I was growing up (between ages 5-15)". A continuous total family relationships variable was created by taking the sum across items comprising the three subscales, with conflict items being reversed scored so that higher values indicate less conflict.

2.3.4.4. Family dysfunction: The Risky Families Questionnaire (Taylor, Way, \& Seeman, 2011) was used to assess the extent of family dysfunction during childhood and adolescence. Using a 5-point frequency scale $(1=$ not at all to $5=$ very often $)$ respondents rank the extent to which their family was lacking in nurturance and affection (how often did a parent or other adult make you feel loved, supported, and cared for?), characterized by overt conflict and aggression (how often was there quarreling, arguing, or shouting between your parents?), chaotic (was the household chaotic and disorganized?), and/or abusive (how often did a parent or other adult push, grab, shove, or slap you?). For all items, participants were instructed to respond with reference to -When I was growing up (between ages 5-15)". Participants' responses to the 10-item scale were summed to create a continuous total score.

2.3.4.5. Parental bonding: A modified version of the Parental Bonding Instrument (PBI; Parker, Tupling, \& Brown, 1979) was used to assess the extent to which participants had 
established an emotional bond with their parents. The scale measures two components of the parent-child relationship: demonstrations of caring (my parents were affectionate to me) and parental overprotection (my parents tried to control everything I did). Using a 4-point rating scale ( 1 = very like to 4 very unlike), respondents indicate the degree to which a series of phrases describing parental behaviors accurately reflect those enacted by their own parents during the first 15 years of my life. Prior to analysis, items were scaled such that higher total scores reflect optimal bonding, which is considered to be high levels of caring and low overprotection. The modified version of the PBI that was administered in the present study contained 12 of the 25 items that comprise the original scale. Included items were chosen based on the results of a factor analysis that was published in the source article (Parker et al., 1979). In the present sample, the reduced scale shows good internal reliability (Cronbach's $a=.80)$.

\subsection{Adult Psychological Well-Being}

Two measures were used to assess current psychological well-being: perceived stress and emotional stability (inverse of neuroticism). Perceived stress was assessed using the Perceived Stress Scale (PSS; Cohen, Kamarck, \& Mermelstein, 1983), a 10-item instrument measuring the extent to which individuals perceive the past month as being unpredictable, uncontrollable, and overwhelming. Emotional stability was assessed using 8 items from the International Personality Item Pool: get stressed out easily; worry about things; am easily disturbed; get upset easily; change my mood a lot; have frequent mood swings; get irritated easily; often feel blue (Goldberg, et al., 2006). All items are reverse-scored so that higher values indicate greater emotional stability.

\subsection{Antibody to cytomegalovirus (CMV)}

Plasma from baseline blood samples was assayed for IgG antibodies against CMV using a commercially available enzyme-linked immunosorbent assay (ELISA) (Biocheck, Inc., CA, USA) according to manufacturer instructions. Assay results were used to derive a CMVspecific IgG antibody index, which provides a relative measure of CMV antibody levels that is proportional to but not a direct measure of levels. Index values of 0.90 and lower indicate negative CMV serostatus; values of 1.00 or greater indicate positive serostatus. Among participants determined to be seropositive for CMV, continuous index values were used as a proxy measure for CMV antibody levels.

\subsection{Control variables}

Covariates were chosen based both on their likely associations with childhood sociodemographic variables and with prior research showing them to be related to CMV seroprevalence (Dowd, Palermo, \& Aiello, 2012; Staras et al., 2006). Demographic variables included self-reported age, sex, race/ethnicity, education, and household income. For race/ ethnicity, participants selected from white, black, Native American, Asian/Pacific Islander, Hispanic/Latino, or other. Due to the small number of participants endorsing nonblack minority categories, a dichotomous variable $(0=$ white, $1=$ all others $)$ was used to represent race in analyses. Education was represented by a 9 category variable ranging from did not finish high school to doctoral degree; and annual household income by a 13-category 
variable ranging from less than $\$ 5000$ to $\$ 150,000$ or more. For analysis, a current adult SES index variable was created by combining standardized education and income values (zscores), and then taking the square-root of that quantity to eliminate skew. Additional covariates included body mass index (BMI; $\mathrm{kg} / \mathrm{m}^{2}$ ) computed from laboratory measurements of participants' height and weight, and self-reported current smoker status (1 $=$ current daily smoker; $0=$ former/never smoker).

\subsection{Statistical analysis}

All statistical analyses were performed using IBM SPSS Version 21 (IBM Corp., Armonk, NY) and all tests were two-tailed. T-tests were used to compare means of continuous predictors across CMV serostatus groups, and chi-square tests were used to examine differences in the relative prevalence of dichotomous predictors between groups. Pearson correlations were used to examine associations among continuous predictor variables. Multivariable logistic regression was used for examining the association of childhood environments with adult CMV serostatus, with results reported as odds ratios (ORs) and 95\% confidence intervals (CIs). Multivariable linear regression was used to examine associations of childhood environments with CMV antibody concentrations among those determined to be seropositive. For these analyses, the unstandardized regression coefficient (B), standard error (SE) of B, standardized coefficient ( $\beta$ ), change in the multiple correlation coefficient $\left(\Delta R^{2}\right)$, and $p$-value are reported. The six control variables described in the previous section (age, sex, race, SES, BMI, and smoker status) were included in all of the main analyses.

Both concurrent and retrospective evaluations of the self or one's environment are thought to be influenced by psychological states. Indicators of psychological well-being, particularly stress and neuroticism, have been associated with more negative reporting (Costa \& McCrae, 1985). Moreover, psychological stress and negative emotions have been associated with various indices of immune function (Glaser \& Kiecolt-Glaser, 2005). To rule out the possibility that current adult psychological well-being might account for any found associations between childhood environments and CMV serostatus and antibody levels, a set of exploratory analyses were conducted that included current adult perceived stress and emotional stability (the inverse of neuroticism) in the above-described models.

\section{Results}

\subsection{Sample characteristics}

Approximately $39 \%$ ( $n=53$ ) of the sample was seropositive for antibody to CMV. As indicated by Table 1, likelihood of being CMV seropositive increased with age and BMI, was greater among women, blacks, and smokers, and was lower among whites.

\subsection{Relation of childhood environments with adult CMV serostatus}

Table 2 presents descriptive statistics for childhood environment variables separately by CMV serostatus. The table also displays results of separate multivariable logistic regression analyses (expressed as odds ratios [OR] and 95\% confidence intervals [CI]) examining the association of each childhood environment variable with adult CMV serostatus. 
Characteristics of the childhood neighborhood environment consistently predicted CMV serostatus, with each additional report of having lived in a city (sum of reports for ages 5 , 10 , and 15 years) being associated with a $55 \%$ increase in the odds of being CMV+ as an adult; each 1-point increase in the measure of poor childhood neighborhood physical conditions being associated with a $65 \%$ increase in the odds of CMV seropositivity; and each 1-point increase in the measure of low childhood neighborhood safety being associated with a $130 \%$ increase. The single exception was neighborhood social environment, which was unrelated to CMV serostatus. Family SES also was related to CMV serostatus, with each additional year-between the ages of 1 and 18 years, of living in a home that was owned by one's parents being associated with a 7\% decrease in the odds of being CMV+ as an adult. Of the two residential exposure variables, only parental smoking was associated with CMV, with those participants who reported either parent ever having been a smoker during their childhood having more than twice the odds of being CMV+ as adults relative to those whose parents never smoked. By comparison, none of the family environment variables were related to CMV serostatus.

Family SES and the three neighborhood environment variables that were associated with CMV serostatus were moderately correlated with one another, ranging from $r=-0.30$ for the association of family SES with neighborhood safety to $r=0.80$ for neighborhood physical conditions and safety (all $p<0.001$ ). Parental smoking also was associated with these variables, such that those who reported having a parent who smoked also reported more ages living in a city $(t[138]=3.41)$, worse neighborhood physical conditions $(t[138]=$ $3.76)$, lower neighborhood safety $(t[138]=3.31)$, and fewer years living in a parent-owned home $(t[114]=3.39$; all $p<0.001)$. To determine the relative importance of each of the significant predictors, neighborhood physical conditions, neighborhood safety, number of ages reported living in a city, and parental smoking were entered into a forward stepwise logistic regression analysis that included all of the standard control variables. Parental smoking emerged as the best predictor of CMV serostatus $\left(\mathrm{chi}^{2}=8.98, \mathrm{df}=1, p<0.005\right)$, with only neighborhood physical conditions explaining additional variance in serostatus after the effect of parental smoking was accounted for $\left(\mathrm{chi}^{2}=5.86, \mathrm{df}=1, p<0.02\right)$.

\subsection{Relation of childhood environments with CMV antibody levels among seropositive adults}

Table 3 presents results of separate multivariable linear regression analyses examining the association of each childhood environment variable with CMV antibody levels among those participants who had been identified as being CMV+ as adults $(n=53)$. In contrast to the findings for serostatus, neither childhood neighborhood, family SES, nor residential exposures were related to CMV antibody levels among the seropositive subset. However, with the exception of parental divorce, all family environment variables were associated with CMV antibody levels in the expected directions. Specifically, greater family warmth, greater family harmony, lower family dysfunction, and closer to optimal parental bonding each was associated with lower antibody levels among CMV+ participants.

The four family environment variables that were associated with CMV antibody levels were highly correlated with one another, ranging from $r=0.57$ for the association of family 
warmth with parental bonding to $r=-0.86$ for the association of family harmony with dysfunction (all $p<0.001$ ). To determine the relative importance of each of the significant predictors, warmth, harmony, dysfunction, and parental bonding were entered into a forward stepwise multiple regression analysis that included all of the standard control variables. Family warmth emerged as the best predictor of antibody levels $(F[1,44]=11.23, p=$ 0.002 ), with no other family relationship variables entering the model.

\subsection{Adult psychological well-being as a possible explanation for the association of childhood environments with CMV serostatus and antibody levels}

To rule out the possibility that current adult psychological factors might account for the associations between childhood environments and CMV serostatus and antibody levels, 2 sets of exploratory analyses were conducted that included control for either of two markers of psychological well-being (a) current adult perceived stress or (b) emotional stability (the inverse of neuroticism).

3.4.1. Childhood neighborhood and residential environments, family SES, and CMV serostatus-Greater current adult perceived stress was associated with having been exposed to poorer childhood neighborhood physical conditions $(r=0.18, p=.03)$. However, neither perceived stress nor emotional stability was related to any of the remaining childhood environment variables that were associated with adult CMV serostatus (all $p$ > $0.10)$. Further, neither adult psychological well-being variable was associated with CMV infections (perceived stress, $\mathrm{OR}=1.02,95 \% \mathrm{CI}=0.95,1.09, p=0.66$; emotional stability, $\mathrm{OR}=1.04,95 \% \mathrm{CI}=0.99,1.09, p=0.14)$; and when each was added in separate analyses to the significant models presented in Table 2 , the outcomes were virtually unchanged (see Supplemental Table S1).

\subsubsection{Childhood family environment and CMV antibody levels in seropositive} participants-Among the participants who were seropositive for CMV, emotional stability was associated with all four childhood family environment variables that were related to antibody levels in the direction of greater stability being associated with a more favorable environment (family warmth, $r=0.37$; harmony, $r=0.44$; dysfunction, $r=-0.43$; parental bonding, $r=0.43$; all $p<0.001$ ). By comparison, adult perceived stress was unrelated to childhood family environment (all $p>0.17$ ). Neither perceived stress nor emotional stability was related to antibody levels among seropositive persons (perceived stress, $\mathrm{B}=0.003, \mathrm{SE}=0.01, \beta=0.04, p=0.79$; emotional stability, $\mathrm{B}=-0.01, \mathrm{SE}=0.01, \beta$ $=-0.15, p=0.25$ ). Although the results were unchanged when perceived stress was added to the significant models presented in Table 3 , the associations of childhood family harmony and family dysfunction with antibody levels no longer met the $\mathrm{p}<0.05$ criterion. However, for neither variable was the size of the association appreciably changed (harmony, $\mathrm{B}=$ $-0.011, \mathrm{SE}=0.01, p=0.08$; dysfunction, $\mathrm{B}=0.009, \mathrm{SE}=0.005, p=0.11$ ). Models of the associations of childhood family warmth and parental bonding were unchanged by the addition of emotional stability (see Supplemental Table S2). 


\section{Discussion}

In the present sample of 140 healthy men and women, less favorable socioeconomic conditions during childhood were associated with increased likelihood of having been infected with CMV, independent of age, sex, race, BMI, current adult SES, and current smoking status. The present findings are consistent with existing research among children and adolescents that family SES predicts concurrent CMV serostatus (Staras, Flanders, Dollard, \& et al., 2008; Dowd, Aiello, \& Alley, 2009; Dowd et al., 2012). They also extend the child and adolescent data by providing evidence to suggest that early life exposures to conditions that become increasingly prevalent at lower levels of SES—-specifically, poor neighborhood physical conditions, low neighborhood safety, and having at least one parent who smoked, also may play an important role in establishing one's risk of becoming infected with CMV. This elevated risk could result from increased susceptibility to infection and/or increased exposure to the virus. One might speculate that parental smoking may have a greater influence on the former, whereas neighborhood environmental factors may play a larger role in the latter. Given the independent contributions of parental smoking and neighborhood physical environment to the odds of being CMV+, it seems likely that both susceptibility and exposure influence risk for infection.

In regard to differential exposure to CMV by neighborhood environment, it is possible that children living in unsafe or poorly-maintained neighborhoods are at higher risk of being exposed to CMV because they spend more time inside either their own home or the homes of neighboring children. Different than in healthy adults, young children who have been infected with CMV continue to shed the virus for several months following initial exposure (Pass, 2002). It seems likely therefore that close proximity to children who are infected with $\mathrm{CMV}$ - or who have infected siblings, are more likely to become infected.

The analysis of CMV antibodies among the seropositive subset of the sample revealed reliable effects for family relationships during childhood. Specifically, greater family dysfunction, less family harmony, low levels of parental warmth, and less than optimal parental bonding each were associated with higher levels of CMV antibody, with parental warmth accounting for most of the variance. As above, all associations were independent of age, sex, race, BMI, current adult SES, and current smoking status. These results replicate those observed among breast cancer survivors (Fagundes et al., 2013) wherein childhood family adversity was associated with higher antibody against latent viruses during adulthood as well as extend them to a healthy sample comprised of both women and men.

Despite evidence showing CMV antibody titers in both children and adults vary inversely with concurrent SES (Dowd et al., 2012; Dowd et al., 2009), here we found only a trend ( $p=$ 0.13) toward more years of parental home ownership being associated with lower CMV antibody levels in adulthood. It is possible that this non-significant finding is due to the substantial reduction in sample size caused by the large number of individuals within the subset who had missing parental home ownership data.

The relatively small sample size overall $(n=140)$ is a limitation to the present study because it does not provide sufficient power to test for potential moderators. For example, there is 
evidence to suggest that the association of traumatic life events with EBV antibody titers is stronger among female relative to male adolescents (McDade, et al., 2000). Future studies might examine whether sex similarly moderates the association of childhood environments with reactivation of latent viruses in adulthood. Also, the parent study included strict inclusion and exclusion criteria, thus resulting in a sample free of chronic disease and regular medication use. Accordingly, the present findings among a relatively small and healthy sample may be limited in the extent to which they generalize to the larger population.

Another limitation of the present study is the overall retrospective design. Because CMV serostatus was assessed during adulthood, it cannot be determined when CMV+ participants became infected with the virus. However, approximately $42 \%$ of children and adolescents have been infected with CMV by 19 years of age (Staras et al., 2008). Given that the seroprevalence in the present sample was 39\%, it seems likely that a substantial number of $\mathrm{CMV}+$ participants had become infected prior to reaching adulthood. A potentially more problematic component of the retrospective design is that childhood environmental exposures also were assessed during adulthood, thus subjecting these data to the effects of retrospective bias. To rule out the possibility that reports of childhood neighborhood quality and family relationships may have been influenced by current adult psychological wellbeing, we conducted two additional sets of analyses that controlled for emotional stability (the inverse of neuroticism), and current perceived stress levels, respectively. These additional controls did not change the findings appreciably, save for reductions in significance for the associations of childhood family harmony and family dysfunction with CMV antibody levels when emotional stability was added to the respective models. As the sizes of the associations were virtually unchanged, the drop in significance likely was due to the loss of a degree of freedom rather than to a confounding effect.

One hypothesis that has been offered to explain the link between psychosocial factors and elevated levels of antibody against latent viruses is that increased activation of the biological stress axis has a down-regulatory effect on cell-mediated immunity, thus permitting viral reactivation (Glaser et al., 1985; Glaser \& Kiecolt-Glaser, 1997). That virus-specific antibody levels increase with increasing viral load is well-supported (Besson, et al., 2006; Kuo, Wu, Ho, Chen, Liu, \& Lu, 2008; van Zanten, et al., 1995). However, repeated infection with different strains of the same virus (i.e., super-infection) also may raise $\mathrm{IgG}$ levels (Novak, et al., 2008; Ross, Arora, Novak, Fowler, Britt, \& Boppana, 2010). Because the psychological stress factors associated with CMV-IgG levels were not related to CMV infection, and the physical environmental factors associated with CMV serostatus likewise were unrelated to antibody levels, our results are more consistent with a mechanism involving viral reactivation than super-infection. We offer this interpretation cautiously, however, because initial infection could have occurred during adulthood, and because we do not know the extent to which reactivation processes might have begun during childhood.

Overall, the present findings contribute further evidence in support of early life experiences acting as significant modifiers of immunocompetence, in particular as it pertains to CMV infection and latency. In addition to replicating the findings of previous CMV serostatus research conducted in children and adolescents, the present data extend the earlier work by 
demonstrating that the effects of family SES on risk for CMV infection persist beyond early life, and act independently of adult socioeconomic factors. These results also elaborate upon the as yet small literature demonstrating an association between early life adversity and reactivation of latent viruses in adulthood. Finally, the present data provide suggestive evidence that different types of childhood adversity may be associated with differential effects on CMV infection and latency. Specifically, low SES and poor neighborhood and residential environmental conditions may predispose to infection, whereas family dysfunction, lack of parental warmth and suboptimal parental bonding may lead to future susceptibility to viral reactivation. Because of the substantial intercorrelations among the measures comprising these two sets of predictors, it seems unlikely that any single variable constitutes a uniquely important toxic exposure. Rather, each set of variables might be considered jointly as representing the broader constructs of material and psychosocial adversity.

\section{Supplementary Material}

Refer to Web version on PubMed Central for supplementary material.

\section{Acknowledgments}

This research was supported by the National Institute of Allergy and Infectious Diseases (AI066367) and the National Center for Complementary and Alternative Medicine (AT006694) and through supplemental support from the John D. and Catherine T. MacArthur Foundation's Research Network on Socioeconomic Status and Health, the Eberly Foundation, the Hamburg Fellowship, and National Institutes of Health funding to the University of Pittsburgh Clinical and Translational Science Institute (UL1 RR024153 and UL1TR000005).

We are indebted to Ellen Conser, James Seroky, Julie Banks and Wesley Barnhart for their assistance in data collection.

\section{References}

Besson C, Amiel C, Le-Pendeven C, Brice P, Ferme C, Carde P, Hermine O, Raphael M, Abel L, Nicolas JC. Positive correlation between Epstein-Barr virus, viral load and anti-viral capsid immunoglobulin $\mathrm{G}$ titers determined for Hodgkin's lymphoma patients and their relatives. J Clin Microbiol. 2006; 44:47-50. [PubMed: 16390946]

Carroll J, Cohen S, Marsland A. Early childhood socioeconomic status is associated with circulating interleukin-6 among mid-life adults. Brain Behav Immun. 2011; 25:1468-1474. [PubMed: 21672624]

Chidrawar S, Khan N, Wei W, McLarnon A, Smith N, Nayak L, Moss P. Cytomegalovirusseropositivity has a profound influence on the magnitude of major lymphoid subsets within healthy individuals. Clin Exp Immunol. 2009; 155:423-432. [PubMed: 19220832]

Cohen, S. SC Places You've Lived Interview. 2010a. Retrieved from http://www.psy.cmu.edu/ $\sim$ scohen

Cohen, S. SC Childhood Interview. 2010b. Retrieved from http://www.psy.cmu.edu:16080 scohen/

Cohen S, Doyle W, Turner R, Alper C, Skoner D. Childhood socioeconomic status and host resistance to infectious illness in adulthood. Psychosom Med. 2004; 66:553-558. [PubMed: 15272102]

Cohen, S.; Janicki-Deverts, D.; Chen, E.; Matthews, K. Childhood socioeconomic status and adult health. In: Adler, N.; Stewart, J., editors. The biology of disadvantage. New York: Annals of the New York Academy of Sciences; 2010. p. 37-55.

Cohen S, Janicki-Deverts D, Turner R, Marsland A, Casselbrant M, Li-Korotky HS, Epel E, Doyle W. Childhood socioeconomic status, telomere length, and susceptibility to upper respiratory infection. Brain Behav Immun. 2013; 34:31-38. [PubMed: 23845919] 
Cohen S, Kamarck T, Mermelstein R. A global measure of perceived stress. Journal of Health and Social Behavior. 1983; 24:385-396. [PubMed: 6668417]

Costa PT Jr, McCrae RR. Hypochondriasis, neuroticism, and aging: When are somatic complaints unfounded? Am Psychol. 1985; 40:19-28. [PubMed: 3977166]

Danese A, Parlante C, Caspi A, Taylor A, Poulton R. Childhood maltreatment predicts adult inflammation in a life-course study. Proc Natl Acad Sci. 2007; 104(4):1319-1324. [PubMed: 17229839]

Dowd J, Aiello A, Alley D. Socioeconomic disparities in the seroprevalence of cytomegalovirus infection in the US population: NHANES III. Epidemiol Infect. 2009; 137:58-65. [PubMed: 18413004]

Dowd J, Bosch J, Steptoe A, Blackburn E, Lin J, Rees-Clayton E, Aiello A. Cytomegalovirus is associated with reduced telomerase activity in the Whitehall II cohort. Exp Gerontol. 2013; 48:385-390. [PubMed: 23403382]

Dowd J, Palermo T, Aiello A. Family poverty is associated with cytomegalovirus antibody titers in U.S. children. Health Psychol. 2012; 31(1):5-10. [PubMed: 21895372]

Dowd J, Palermo T, Chyu L, Adam E, McDade T. Re: Childhood adversity and cell-mediated immunity in young adulthood. Brain Behav Immun. 2013; 34:176. [PubMed: 23938352]

Fagundes C, Glaser R, Johnson S, Andridge R, Yang E, Di Gregorio M, Chen M. Basal cell carcinoma: Stressful life events and the tumor environment. Arch Gen Psychiatry. 2012; 69(6): 618-626. [PubMed: 22664550]

Fagundes C, Glaser R, Malarkey W, Kiecolt-Glaser J. Childhood adversity and herpesvirus latency in breast cancer survivors. Health Psychol. 2013; 32(3):337-344. [PubMed: 22746260]

Freeman R. The "indirect" effects of cytomegalovirus infection. Am J Transplant. 2009; 9:2453-2458. [PubMed: 19843027]

Gkrania-Klotsas E, Langenberg C, Sharp S, Luben R, Khaw K, Wareham N. Seropositivity and higher immunoglobulin $\mathrm{G}$ antibody levels against cytomegalovirus are associated with mortality in the population-based European prospective investigation of Cancer-Norfolk cohort. Clin Infect Dis. 2013; 56(10):1421-1427. [PubMed: 23442763]

Glaser R, Kiecolt-Glaser J. Chronic stress moduates the virus-specific immune response to latent herpes simplex virus 1. Ann Behav Med. 1997; 19(2):78-82. [PubMed: 9603681]

Glaser R, Kiecolt-Glaser J. Stress-induced immune dysfunction: Implications for health. Nat Rev Immunol. 2005; 5(3):243-251. [PubMed: 15738954]

Goldberg L, Johnson J, Eber H, Hogan R, Ashton M, Cloninger C, Gough HG. The International Personality Item Pool and the future of public-domain personality measures. J Res Pers. 2006; 40:84-96.

IBM Corp. IBM SPSS Statistics for Windows, Version 21.0. Armonk, NY: IBM Corp; 2012.

Janusek L, Tell D, Albuquerque K, Mathews H. Childhood adversity increases vulnerability for behavioral symptoms and immune dysregulation in women with breast cancer. Brain Behav Immun. 2013; 30:S149-S162. [PubMed: 22659062]

Kiecolt-Glaser J, Gouin J, Weng N, Malarkey W, Beversdorf D, Glaser R. Childhood adversity heightens the impact of later-life caregiving stress on telomere length and inflammation. Psychosom Med. 2011; 73(1):16-22. [PubMed: 21148804]

Kuo C, Wu C, Ho H, Chen C, Liu S, Lu Y. Detection of cytomegalovirus reactivation in cancer patients receiving chemotherapy. Clin Microbiol Infect. 2008; 14:221-227. [PubMed: 18070129]

McDade T, Stallings J, Angold A, Costello E, Burleson M, Cacioppo J, Glaser R, Worthman CM. Epstein-Barr virus antibodies in whole blood spots: A minimally invasive method for assessing an aspect of cell-mediated immunity. Psychosom Med. 2000; 62(4):560-568. [PubMed: 10949102]

McElhaney J, Zhou X, Talbot H, Soethout E, Bleackley R, Granville D, Pawelec G. The unmet need in the elderly: How immunosenescence, CMV infection, co-morbities and frailty are a challenge for the development of more effective influenza vaccines. Vaccine. 2012; 30:2060-2067. [PubMed: 22289511]

Miller G, Chen E, Parker K. Psychological stress in childhood and susceptibility to the chronic diseases of aging: Moving toward a model of behavioral and biological mechanisms. Psychol Bull. 2011; 137(6):959-997. [PubMed: 21787044] 
Moos, R.; Moos, B. Family environment scale manual. Consulting Psychologists Press; 1994.

Nikolich-Zugich J. Ageing and life-long maintenance of T-cell subsets in the face of latent persistent infections. Nat Rev Immunol. 2008; 8:512-522. [PubMed: 18469829]

Novak Z, Ross S, Patro R, Pati S, Kumbla R, Brice S, Boppana S. Cytomegalovirus strain diversity in seropositive women. J Clin Microbiol. 2008; 46:882-886. [PubMed: 18216215]

Parker G, Tupling H, Brown L. A parental bonding instrument. Br J Med Psychol. 1979; 52:1-10.

Pass R. Cytomegalovirus infection. Pediatr Rev. 2002; 23(5):163-170. [PubMed: 11986492]

Phillips J, Marsland A, Flory J, Muldoon M, Cohen S, Manuck S. Parental education is related to Creactive protein among female middle aged community volunteers. Brain Behav Immun. 2009; 23:677-683. [PubMed: 19486653]

Pollitt R, Kaufman J, Rose K, Diez-Roux A, Zeng D, Heiss G. Early-life and adult socioeconomic status and inflammatory risk markers in adulthood. Eur J Epidemiol. 2007; 22(1):55-66. [PubMed: 17225957]

Qiu H, Straat K, Rahbar A, Wan M, Soderberg-Naucler C, Haeggstrom J. Human CMV infection induces 5-lipoxygenase expression and leukotriene B4 production in vascular smooth muscle cells. J Exp Med. 2008; 205:19-24. [PubMed: 18180307]

Ross D, Dollard S, Victor M, Sumartojo E, Cannon M. The epidemiology and prevention of congenital cytomegalovirus infection and disease: Activities of the Centers for Disease Control and Prevention Workgroup. J Womens Health. 2006; 15:224-229.

Ross S, Arora N, Novak Z, Fowler K, Britt W, Boppana S. Cytomegalovirus reinfections in healthy seroimmune women. J Infect Dis. 2010; 201:386-389. [PubMed: 20039807]

Savva G, Pachnio A, Kaul B, Morgan K, Huppert F, Brayne C, Moss PA. Cytomegalovirus infection is associated with increased mortality in the older population. Aging Cell. 2013; 12(3):381-387. [PubMed: 23442093]

Shirtcliff E, Coe C, Pollak S. Childhoood stress is associated with elevated antibody levels to herpes simplex virus type 1. Proc Natl Acad Sci. 2009; 106(8):2963-2967. [PubMed: 19188604]

Simanek A, Dowd J, Pawelec G, Melzer DAD, Aiello A. Seropositivity to cytomegalovirus, inflammation, all-cause and cardiovascular disease-related mortality in the United States. PLoS ONE. 2011; 6(2):e16103. [PubMed: 21379581]

Slopen N, McLaughlin K, Dunn E, Koenen K. Reply to Letter Re: Childhood adversity and cellmediated immunity in young adulthood. Brain Behav Immun. 2013; 34:177-179. [PubMed: 23954376]

Sobel, M. Asymptotic confidence intervals for indirect effects in structural equations models. In: Leinhart, S., editor. Sociological Methodology 1982. San Francisco: Jossey-Bass; 1982. p. 290-312.

Staras S, Dollard S, Radford K, Flanders W, Pass R, Cannon M. Seroprevalence of cytomegalovirus infection in the United States, 1988-1994. Clin Infect Dis. 2006; 43(9):1143-1151. [PubMed: 17029132]

Staras S, Flanders W, Dollard S, Pass R, McGowan J Jr, Cannon M. Cytomegalovirus seroprevalence and childhood sources of infection; A population-based study among pre-adolescents in the United States. J Clin Virol. 2008; 43(3):266-271. [PubMed: 18778968]

Taylor S, Way B, Seeman T. Early adversity and adult health outcomes. Dev Psychopathol. 2011; 23:939-954. [PubMed: 21756443]

Turner J, Campbell J, Edwards K, Howarth L, Pawelec G, Aldred S, Moss P, Drayson M, Burns V, Bosch J. Rudimentary signs of immunosenescence in Cytomegalovirus-seropositive healthy young adults. Age. 2014; 36(1):287-297. [PubMed: 23846127]

van de Berg P, Griffiths S, Yong SL, Macaulay R, Bemelman F, Jackson S, Henson S, ten Berge I, Akbar A, van Lier A. Cytomegalovirus infection reduces telomere length of the circulating T cell pool. J Immunol. 2010; 184:3417-3423. [PubMed: 20176738]

van Zanten J, Harmsen M, van der Giessen M, van der Bij W, Prop J, de Leij L. Humoral immune response against human cytomegalovirus (HCMV)-specific proteins after HCMV infection in lung transplantation as detected with recombinant and naturally occurring proteins. Clin Diagn Lab Immunol. 1995; 2:214-218. [PubMed: 7535179] 


\section{Table 1}

Sample Characteristics ${ }^{a, b}$

\begin{tabular}{lllll}
\hline & Sample $(\boldsymbol{n}=\mathbf{1 4 0})$ & $\mathbf{C M V}-(\boldsymbol{n}=\mathbf{8 7})$ & $\mathbf{C M V +}(\boldsymbol{n}=\mathbf{5 3})$ & $\boldsymbol{p}_{\text {diff }}$ \\
\hline Age (years) & $30.0(10.7)$ & $27.6(8.9)$ & $33.9(12.3)$ & .001 \\
Sex (female) & $41.4(58)$ & $35.6(31)$ & $50.9(27)$ & .07 \\
Race/Ethnicity & & & & .003 \\
$\quad$ White & $63.6(89)$ & $73.6(64)$ & $47.2(25)$ & \\
$\quad$ Black/African-American & $25.7(36)$ & $16.1(14)$ & $41.5(22)$ & \\
$\quad$ Other $c$ & $10.7(15)$ & $10.3(9)$ & $11.3(6)$ & \\
Education & & & & .11 \\
$\quad$ High school or less & $25.0(35)$ & $19.5(17)$ & $34.0(18)$ & \\
$\quad<2$ yrs of college & $26.4(37)$ & $24.1(21)$ & $30.2(16)$ & \\
2 yrs of college + degree & $22.1(31)$ & $25.3(22)$ & $17.0(9)$ & \\
$\quad$ Bachelor's degree & $26.4(37)$ & $31.0(27)$ & $18.9(10)$ & \\
Income (thousand $\$ \mathrm{US})$ & $12.5(2.5,175.0)$ & $12.5(2.5,175.0)$ & $12.5(2.5,175.0)$ & .68 \\
Body mass index $\left(\mathrm{kg} / \mathrm{m}^{2}\right)$ & $26.8(6.32)$ & $26.0(5.6)$ & $28.2(7.2)$ & .04 \\
\hline
\end{tabular}

${ }^{a}$ Presented as mean (standard deviation), \% (n), or median (range).

${ }^{b}$ p-values representing differences between CMV serostatus groups were derived from chi-square analyses for dichotomous and categorical variables and independent samples t-tests for continuous variables. Because the distribution of income was skewed toward lower values, the MannWhitney $U$ test was used in place of the t statistic.

${ }^{c}$ Includes Native American, $1.4 \%(\mathrm{n}=2)$; Asian, $2.9 \%(\mathrm{n}=4)$; Hispanic, $1.4 \%(\mathrm{n}=2)$; other, $5 \%(\mathrm{n}=7)$ 


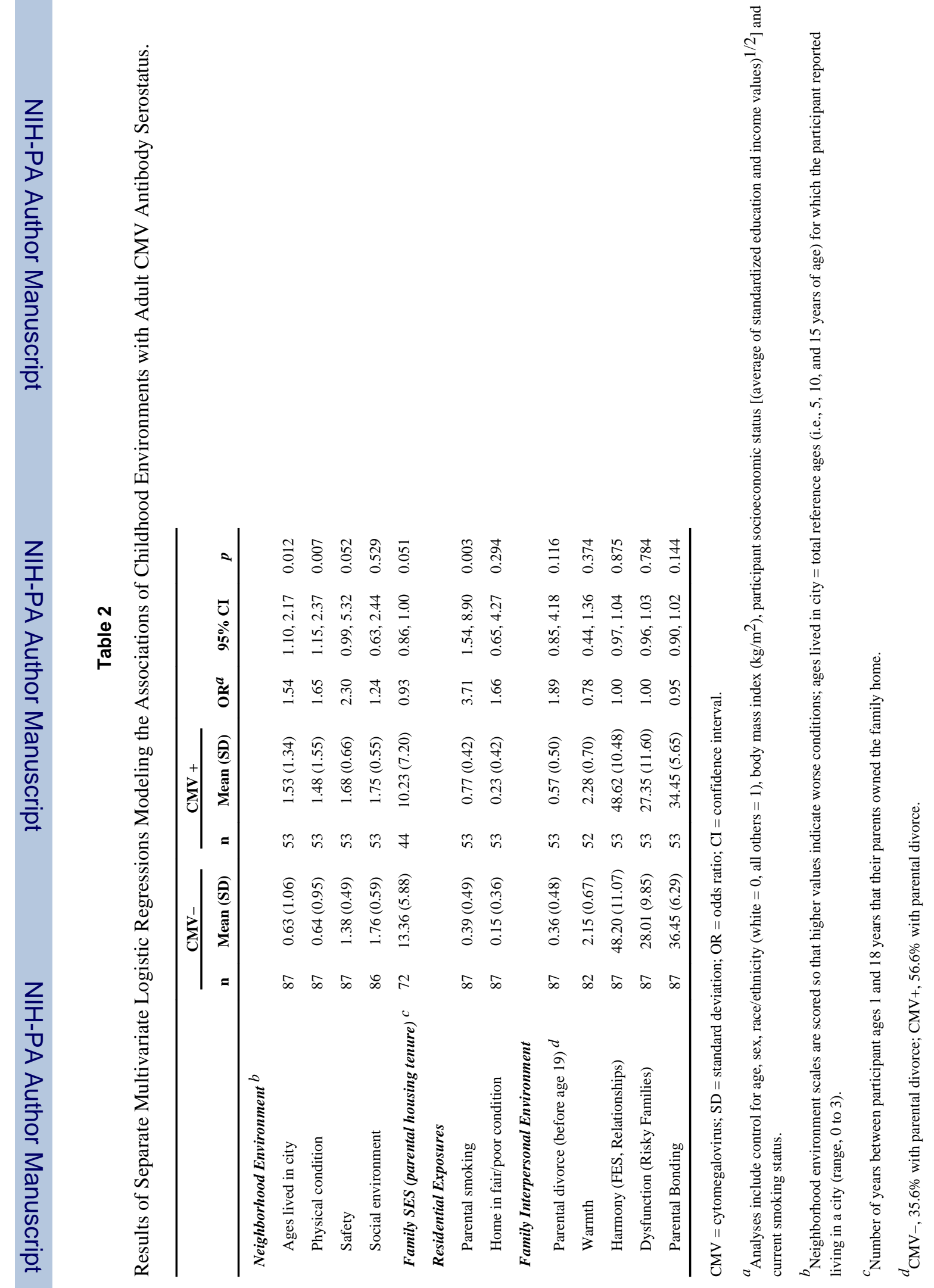




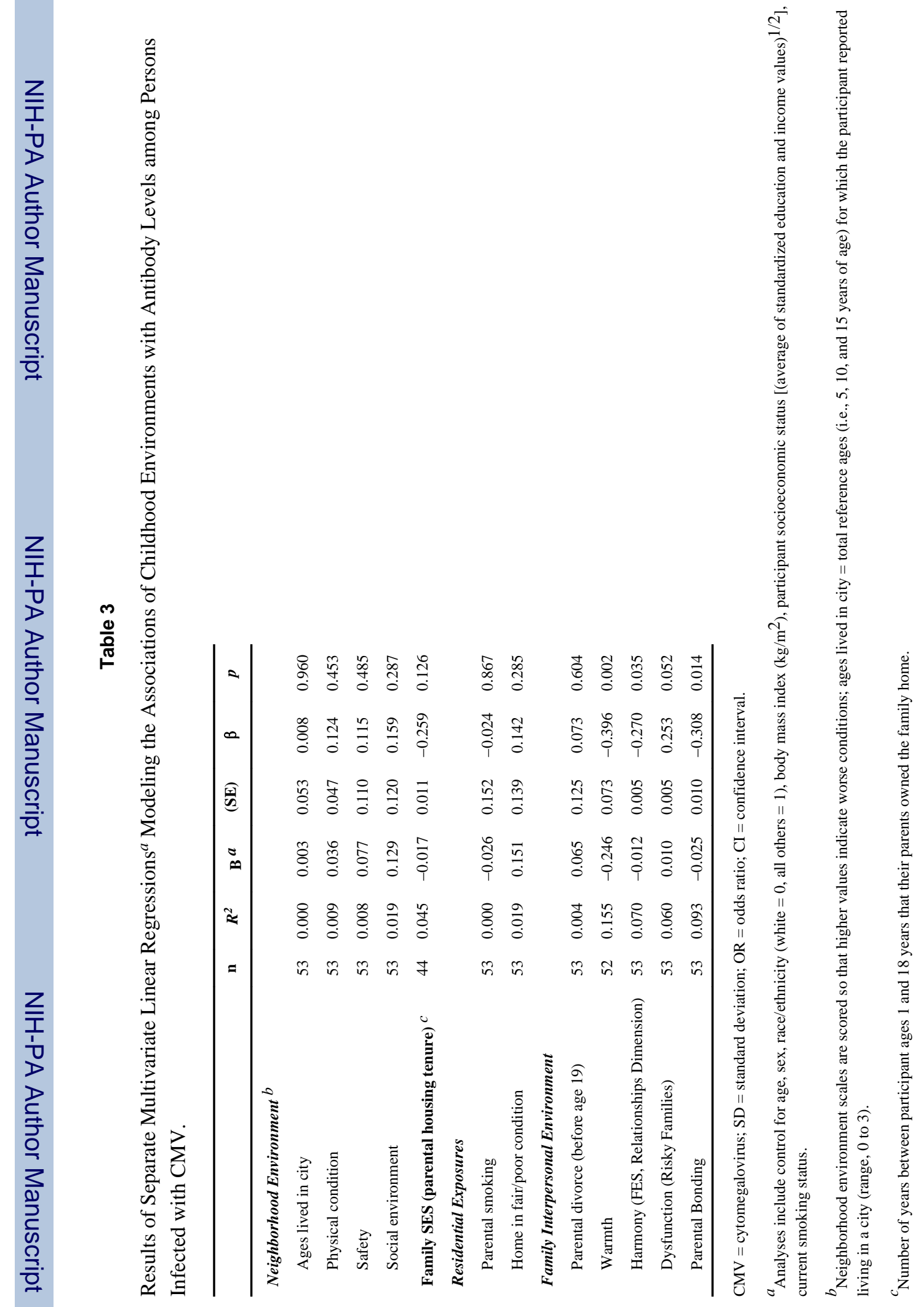

